\title{
Energy Loss Spectroscopy and Electron Microscopy of Photoluminescent p-type Porous Silicon Treated with $\mathrm{NaOH}$ and $\mathrm{NH}_{3}$ Solutions
}

\author{
Minghui Song, Yingda Yu, Yoshio Fukuda, and Kazuo Furuya \\ National Institute for Materials Science, 3-13 Sakura, Tsukuba, Ibaraki 305-0003 JAPAN
}

Porous silicon (PS) formed electrochemically on crystalline silicon at room temperature is known to be visibly photoluminescent [1], and is thought to be a prospective material for optoelectronic devices. Several models have been proposed for the mechanism of photoluminescence. However, little agreement has been attained yet. It is known that the PL is sensitive to both chemical components and microstructures of PS layer. To clarify the effect of microstructure and chemical components of PS on PL is definitely helpful to understand the mechanism of PL of PS. In the present work, electrochemically formed PS on p-type silicon wafers were etched by $\mathrm{NaOH}$ and $\mathrm{NH}_{3}$ hydro-solutions, in order to study the relation between PL and Si-oxide layer on the surface of the PS layer. The change in PL of the treated specimens has been correlated with the changes in local chemical states and microstructure of PS layer with EELS and TEM.

PS samples were prepared by anodizing p-type $\mathrm{Si}(100)$ wafers with resistivity of 0.04-0.06 $\Omega \mathrm{cm}$. Anodization was carried out in a solution of $50 \mathrm{wt} \% \mathrm{HF}: \mathrm{C}_{2} \mathrm{H}_{5} \mathrm{OH}=1: 1$ at room temperature for 10 seconds by applying a current density of $350 \mathrm{~mA} \mathrm{~cm}$. The specimens were then etched using $0.05 \% \mathrm{NaOH}$ hydro-solution from 5 to 60 seconds and $0.14 \% \mathrm{NH}_{3}$ hydro-solution from 1 to 45 minutes at room temperature. The PL spectra were obtained using a photoluminescence-lifetime measurement system with a streak camera (Hama-photo C4780). The local chemical states and microstructure of as-prepared and solution treated PS were analyzed and observed using a JEM-ARM1000 TEM, to which a post-column parallel detection EELS system is attached under the camera chamber.

As-prepared PS layers were shown to be photoluminescent with a peak wavelength around $640 \mathrm{~nm}$. The $\mathrm{NaOH}$ solution etching resulted in a decrease of PL intensity as shown in figure 1. For $\mathrm{NH}_{3}$ treated specimens, the PL around $640 \mathrm{~nm}$ decreased after etching for 1 minute. Further etching up to 10 minutes increased the PL but in a shorter wavelength range. The increased PL then decreased again after 45 minutes etching. The spectra of the PL are shown in figure 2. TEM observation revealed that the PS layer structurally consists of so called a sponge-like part near the surface of the PS and a tree-like structure region in the inner part of the PS layer [2]. The sponge-like layer was apparently reduced by the $\mathrm{NaOH}$ etching, while the tree-like structure remained almost unchanged. For the $\mathrm{NH}_{3}$ treated specimens, TEM observation revealed that the sponge-like layer on surface of PS was resolved by 1-minute etching, and that further treatment up to 10 minutes formed the new sponge-like layer on the surface as shown in figure 3. The 45-minutes treatment resolved the sponge-like layer again. The decrease and increase of the sponge-like layer correlated to the decrease and increase of PL, respectively. The near edge EELS spectra (ELNES) of Si- $\mathrm{L}_{2,3}$ of PS layers were obtained. The $\mathrm{Si}^{4+}$ edge at $108 \mathrm{eV}$ corresponding to the chemical bond of $\mathrm{Si}$ in $\mathrm{Si}-\mathrm{O}$ tetragonal and $\mathrm{Si}^{0+}$ edge at $100 \mathrm{eV}$ corresponding to $\mathrm{Si}$ in silicon crystals were analyzed. These edges changed in its shape and relative intensity after the etching. The correlation of PL intensity in wavelength of $640 \pm 20 \mathrm{~nm}$ with the counts ratio $\mathrm{Si}^{4+} / \mathrm{Si}^{0+}$ in EELS spectra for a $\mathrm{NaOH}$ treated specimen is shown in figure 4, which reveals that the decrease of PL after $\mathrm{NaOH}$ etching is consistent with the decrease of $\mathrm{Si}^{4+} / \mathrm{Si}^{0+}$. The present work suggests that the sponge-like structure near the surface of PS contributes to PL much more than the inner structure of PS layer and that the Si-O chemical structure relates to the PL strongly. 


\section{References}

[1] L.A.Balagurov et al, Appl. Phys. Lett., 69 (1996) 2852.

[2] M. Song et al , Micron, 31 (2000) 429.

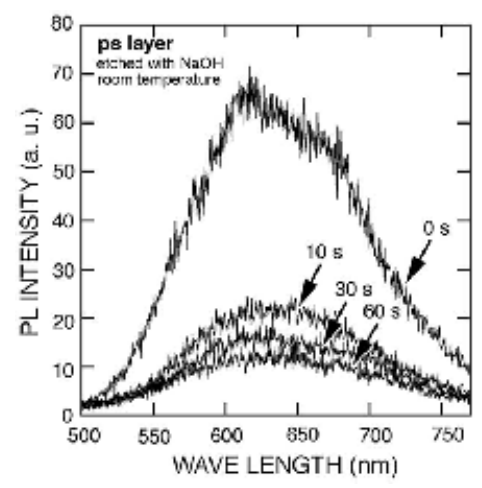

FIG.1

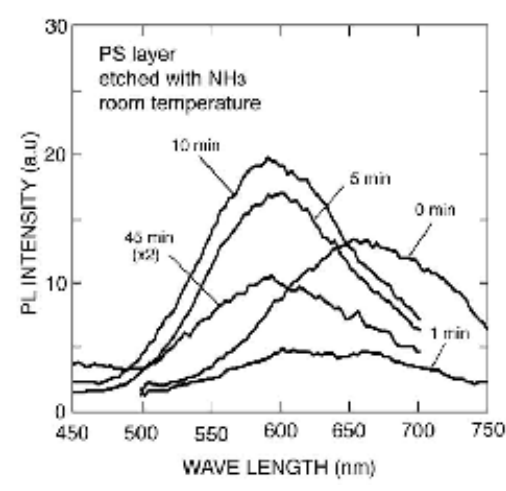

FIG.2

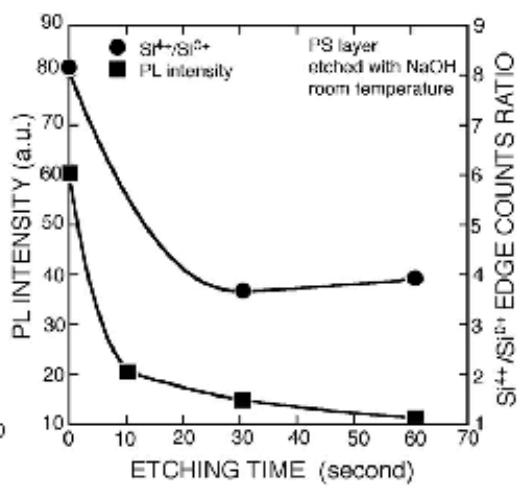

FIG.4
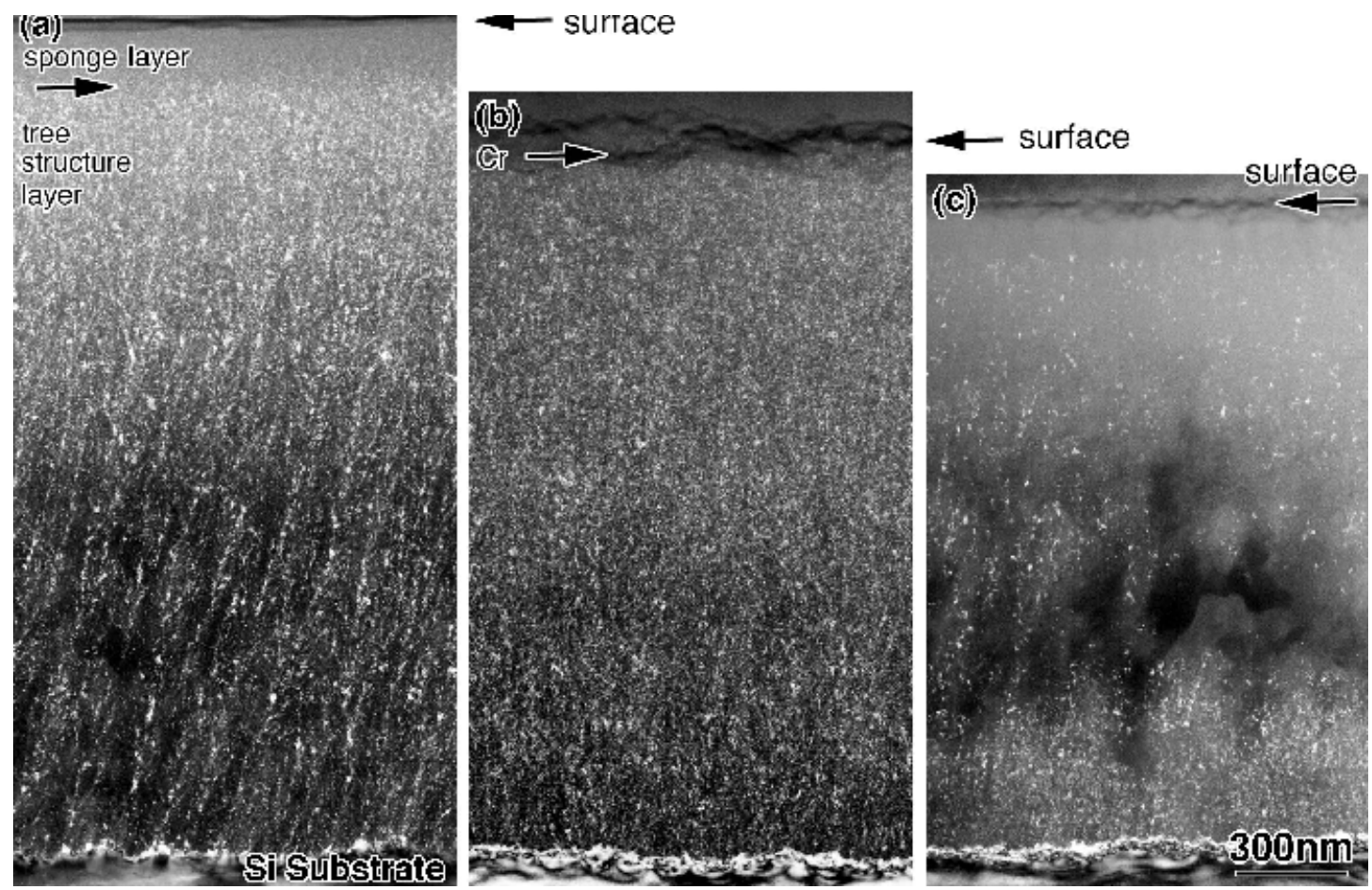

FIG.3

FIG. 1 PL of $\mathrm{NaOH}$ solution treated PS layer formed on low resistivity p-type Si wafers in a current of $350 \mathrm{~mA} \mathrm{~cm}^{-2}$ at room temperature.

FIG. 2 PL of $\mathrm{NH}_{3}$ solution treated PS layer.

FIG. 3. Cross sectional TEM dark field micrographs of PS layer etched with $\mathrm{NH}_{3}$ solution. a) As-prepared, b) etched for 1 minute. c) etched for 5 minutes. Cr layer was coated on the surface to protect PS.

FIG. 4. Correlation of PL intensity with the count ratio of edges $\mathrm{Si}^{4+} / \mathrm{Si}^{0+}$ in EELS spectra of PS layer. 